\title{
Lifestyle Practices and Cardiovascular Disease Mortality in the Elderly: The Leisure World Cohort Study
}

\author{
Annlia Paganini-Hill, 2,3 \\ ${ }^{1}$ Department of Preventive Medicine, Keck School of Medicine, University of Southern California, Los Angeles, CA 90089, USA \\ ${ }^{2}$ Department of Neurology, University of California, Irvine, Irvine, CA 92697, USA \\ ${ }^{3}$ Clinic for Aging Research and Education, 24361 El Toro Road Suite 150, Laguna Woods, CA 92637, USA
}

Correspondence should be addressed to Annlia Paganini-Hill, annliahi@usc.edu

Received 16 September 2010; Accepted 5 December 2010

Academic Editor: Demosthenes Panagiotakos

Copyright (c) 2011 Annlia Paganini-Hill. This is an open access article distributed under the Creative Commons Attribution License, which permits unrestricted use, distribution, and reproduction in any medium, provided the original work is properly cited.

Modifiable behavioral risk factors are major contributing causes of death, but whether the effects are maintained in older adults is uncertain. We explored the association of smoking, alcohol consumption, caffeine intake, physical activity, and body mass index on cardiovascular disease (CVD) mortality in 13,296 older adults and calculated risk estimates using Cox regression analysis in four age groups $(<70,70-74,75-79$, and $80+$ years). The most important factor was current smoking, which increased risk in all age-sex groups. In women, alcohol consumption ( $\leq 3$ drinks/day) was related to decreased (15-30\%) risk in those $<80$ years old; in men, $4+$ drinks/day was associated with reduced (15-30\%) risk. Active 70+ year olds had 20-40\% lower risk. Both underweight and obese women were at increased risk. Lifestyle practices impact CVD death rates in older adults, even those aged $80+$ years. Not smoking, moderate alcohol consumption, physical activity, and normal weight are important health promoters in our aging population.

\section{Introduction}

Cardiovascular disease (CVD) is a leading cause of morbidity and mortality in the United States [1]. It kills one American every 38 seconds and accounts for 1 of every 2.9 deaths, more deaths than any other major cause of death. The 2006 overall death rate due to CVD was 262.5, but the rate increases substantially with age. It is estimated that more than 1 in 3 men and around 1 in 4 women aged 75 and over currently live with the condition. This age group is the fastest growing segment of the US population.

Although CVD is the leading cause of death, modifiable behavioral risk factors are major contributing or actual cause of this mortality [2]. In both younger and older age groups the five key risk factors for CVD are hypertension, high serum cholesterol, diabetes, body mass index, and smoking. Additional lifestyle practices including alcohol consumption and exercise are also related to the disease. The majority of studies of alcohol intake have found J- or U-shaped risk curves with light to moderate drinkers having a lower risk of atherosclerotic CVD than nondrinkers or heavy drinkers
[3]. For physical activity, a dose-response relationship exists between duration and intensity of activity and CVD disease risk, with even relatively low levels of physical activity providing some benefit compared with inactivity [4].

Although these lifestyle practices have substantial health benefits and reduce mortality, few studies have examined their impact in combination and on survival beyond age 75. As part of a prospective cohort study of the effect of modifiable lifestyle practices on longevity and successful aging, we explored the association of smoking, alcohol consumption, caffeine intake, physical activity, and body mass index on CVD mortality in a large cohort (over 13,000) of elderly (median age 74 years) men and women followed for 26 years.

\section{Materials and Methods}

The Leisure World Cohort Study was established in the early 1980s when 13,978 (8877 female and 5101 male) residents of a California retirement community (Leisure World Laguna Hills) completed a postal health survey. The population 
and the cohort are mostly Caucasian, well educated, uppermiddle class, and elderly.

The baseline survey asked about demographic information (birth date, sex, marital status, number of children, height, weight); brief medical history (high blood pressure, angina, heart attack, stroke, diabetes, rheumatoid arthritis, fractures after age 40, cancer, gallbladder surgery, glaucoma, cataract surgery); medication use (hypertensive medication, digitalis, nonprescription pain medication); personal habits (cigarette smoking, exercise, alcohol consumption, vitamin supplement use); usual frequencies of consumption of 58 food (or food groups) that are common sources of dietary vitamin A and C; beverage intake (milk, regular coffee, decaffeinated coffee, black or green tea, and soft drinks).

2.1. Lifestyle Factors. Based on their reported smoking history we classified participants as never, past, or current smokers.

Consumption of alcoholic beverages was asked separately for wine ( 4 oz.), beer $(12 \mathrm{oz}$.), and hard liquor ( $1 \mathrm{oz}$.$) , each$ equivalent to about $1 / 2 \mathrm{oz}$. of alcohol. Response choices for average weekday consumption were never drink, less than 1 , $1,2,3$, and 4 or more drinks. Total alcohol intake per day was calculated by summing the number of drinks consumed of each type [5]. Individuals were then categorized into four groups: $0, \leq 1,2-3$, and $4+$ drinks/day.

We estimated daily caffeine intake by summing the frequency of consumption of each beverage and chocolate multiplied by its average caffeine content ( $\mathrm{mg} /$ standard unit) as $115,3,50,50$, and 6 for regular coffee, decaffeinated coffee, tea, cola soft drinks, and chocolate, respectively [6]. Caffeine intake was categorized as <50, 50-99, 100-199, 200-399, $400+\mathrm{mg} /$ day.

Body mass index (weight $(\mathrm{kg}) /$ height $(\mathrm{m})^{2}$ ) was calculated based on self-reported height and weight at baseline and categorized according to federal guidelines: underweight (<18.5), normal weight (18.5-24.9), overweight (25-29.9), and obese $(30+)[7,8]$.

The amount of time spent on physical activities was ascertained by asking, "On the average weekday, how much time do you spend in the following activities?-active outdoor activities (e.g., swimming, biking, jogging, tennis, vigorous walking), active indoor activities (e.g., exercising, dancing), other outdoor activities (e.g., sightseeing, boating, fishing, golf, gardening, attending sporting events), other indoor activities (e.g., reading, sewing, crafts, board games, pool, attending theater or concerts, performing household chores), watching TV." For each question, the response categories were 0 minutes, 15 minutes, 30 minutes, 1 hour, 2 hours, 3-4 hours, 5-6 hours, 7-8 hours, 9 hours or more per day. The time spent per day in active exercise was calculated by summing the times spent in active outdoor activities and active indoor activities and in other activities by summing the times spent in other outdoor activities and other indoor activities.

2.2. Determination of Outcome. Followup of the cohort is maintained by periodic resurvey and determination of vital status by search of governmental and commercial death indexes and ascertainment of death certificates. Participants were followed to death or December 31, 2007, whichever came first. To date 55 cohort members have been lost to follow up; search of death indices did not reveal that these individuals were deceased. Cause of death was determined from death certificates or by codes provided by the California Department of Vital Statistics. We included as CVD deaths those coded 390-459 in International Classification of Diseases 9 (years 1981-1998) and I00-I99 in International Classification of Diseases 10 (years 1999-2007).

2.3. Statistical Analysis. Hazard ratios (HRs) and $95 \%$ confidence intervals (CIs) were obtained using Cox regression analysis [9]. For the Cox models, chronological age was used as the fundamental time scale with study entry being the age when the survey was completed and the event of interest being age at CVD death. Separate analyses were performed for four age groups $(<70,70-74,75-79$, and $80+$ years $)$ within the two sexes. HRs were calculated for each lifestyle factor adjusted for age (continuous) and then additionally adjusted for the other lifestyle variables plus seven separate histories (no, yes) of hypertension, angina, heart attack, stroke, diabetes, rheumatoid arthritis, and cancer. Statistical analyses were performed using SAS version 9.2 (SAS Institute Inc., Cary, NC). No adjustment in the $P$ values was made for multiple comparisons.

To account for the possibility that recent disease development may have influenced lifestyle practices as well as be related to mortality, we repeated the analyses excluding the first five years of followup.

Previous reports present details of the methods and validity of exposure and outcome data [10-15]. The Institutional Review Boards of the University of Southern California and the University of California, Irvine approved the study.

\section{Results}

After excluding 682 subjects with missing information on the lifestyle factors, we analyzed data on 13,296 subjects (8444 women and 4852 men). At study entry, the participants ranged in age from 44 to 101 years (median: 74 years). By December 31, 2007, the subjects had contributed 180,122 person-years of followup (median: 13.5 years), and 11,929 (7367 women and 4562 men) had died. Age at death ranged from 59 to 108 years (median: 87 years). Over half of all deaths were due to CVD: 4575 women and 2656 men.

Table 1 presents selected characteristics for the participants by sex. Differences between males and females were highly statistically significant $(P<.001)$ for all variables except caffeine $(P<.01)$. Because of these differences as well as the different patterns of smoking (amount and duration), alcohol (type), activities (type), and body-build between men and women and the fact that women live longer on average than men and for comparison with other studies limited to a single sex, we performed separate analyses for men and women.

Tables 2 and 3 show the age-adjusted and multivariableadjusted HRs of CVD mortality for the various lifestyle variables for women and men, respectively. Adjustment 
TABLE 1: Characteristics of the cohort by sex.

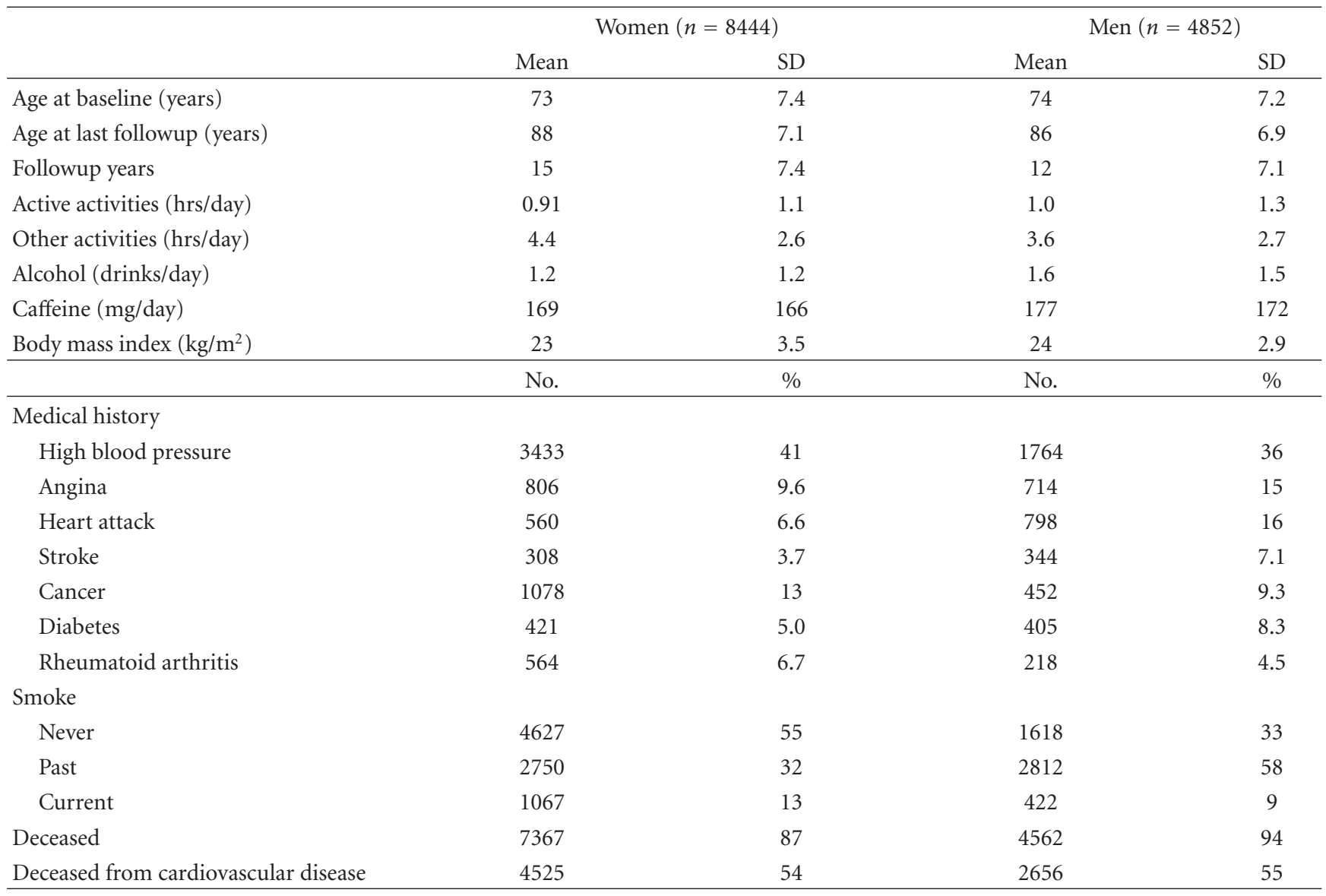

for potential confounders increased the observed HRs for smoking but had limited effect on the others, generally attenuating the observed HRs.

Although caffeine intake showed no consistent effect, the other modifiable factors were related to CVD death. Current smokers had significantly increased (about 40$130 \%)$ risk compared with never smokers in all age-sex groups. In women, alcohol consumption ( $\leq 3$ drinks/day) was related to decreased (about 15-30\%) risk compared with abstainers in all but the oldest age group. In men, $4+$ drinks/day was associated with reduced (about 15-30\%) risk in all but those aged 70-74 years. Women and men aged $70+$ years old who participated in active activities, even as little as $1 / 2$ hour/day, had 20-40\% lower risk of CVD death compared to those who reported no active activities. The risk decreased with increasing time spent in active activities. Participation in other activities was also associated with reduced risk. However, more time in these activities was needed to show the same reduced risk as for active activities. Underweight and obese women in all age groups were at increased risk of CVD death (though not all groups showed statistically significant effects). Notably overweight women had no increased risk. Underweight men also appeared to be at increased risk, though the number of such men was small $(n=81)$. Risk was also increased in overweight men aged $<75$ years compared with their normal weight peers. No effect was seen in older men.

Exclusion of the first five years of followup (including 1826 early deaths) changed the findings slightly. The multivariate-adjusted risk estimates changed by less than 10 percent except for current smokers aged $80+$ years (women 1.46 to 1.28 , men 2.20 to 2.50 ), underweight women aged $70-74$ years (1.29 to 1.13 ), obese women aged $75-79$ years (1.09 to 1.21 ), underweight men (aged $<70$ years, 1.75 to 1.49 ; aged $70-74$ years, 2.76 to 1.84 ; aged $75-79$ years, 1.48 to 1.72 ; and aged $80+$ years, 1.12 to 0.84 ), obese men aged $80+$ years (1.40 to 1.91). HRs for active and other activities in men aged $75+$ years were generally attenuated to 1.0 , and all became nonsignificant.

\section{Discussion}

Our study extends the available literature on the CVD survival benefits of several lifestyle practices to the very old. We confirmed the beneficial effect of not smoking, participating in activities, drinking alcohol, and having a normal body mass index. Each of these was associated with reduced CVD death in our elderly men and women, even those aged 80 years and older. Many of the factors were 


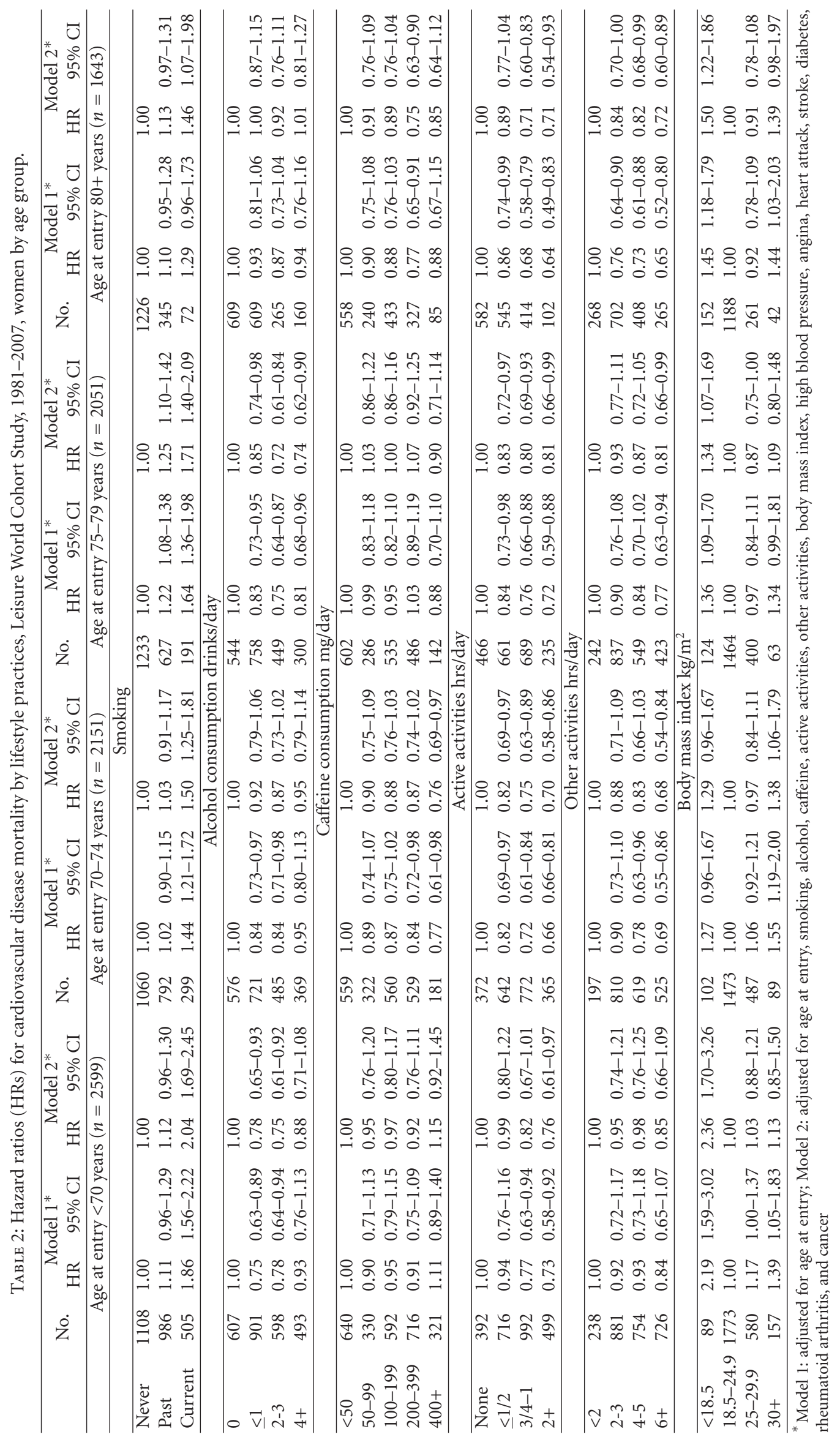




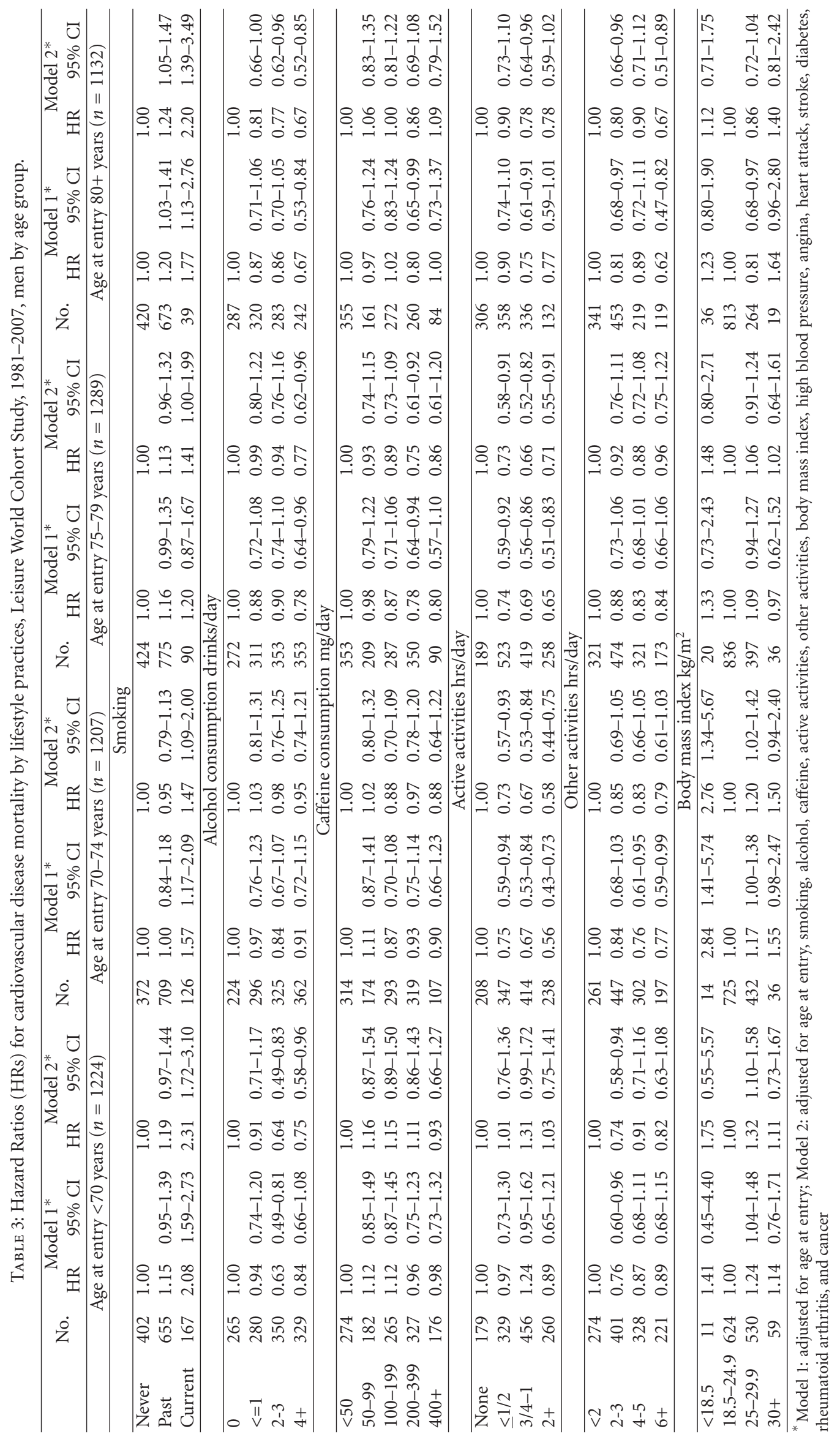


correlated, but each independently predicted risk. The most important single factor was current cigarette smoking.

We previously reported the effects of several of these lifestyle practices on all-cause mortality in this cohort [5, $6,8,15]$. Although not age stratified, results were similar to those found in the present analysis. Smoking increased risk in both men (1.95) and women (1.67). Alcohol intake showed a small beneficial effect (15\% reduction in risk) in both men and women, while a shallow U-shaped association of caffeine intake with mortality was observed in both sexes. The curves for the association of body mass index and allcause mortality were almost identical for men and women with being underweight increasing risk about $50 \%$ and being obese increasing risk $20-25 \%$.

We acknowledge several limitations in our study. The indices of physical activities, alcohol and caffeine intake, and smoking used in this study are crude and self-reported and their reliability and validity were not ascertained. Although our data on other variables are also self-reported, previous studies in our population and others support the reliability of medical history of major chronic disease $[10,13]$ and of self-reported height and weight [10]. Another limitation is that changes over time in all potential risk factors may affect outcome. Additionally, the subjects in our study were mostly white, highly educated, and of middle social-economic class and therefore not representative of the general population. Although this may limit the generalizability of our results, it offers the advantage of reduced potential confounding by race, education, social-economic class, and presumed access to health care. Additionally, although we adjusted for other risk and potential confounding factors, unrecognized and uncontrolled confounders cannot be ruled out in this or any observational study

This cohort has the advantages of population-based prospective design, large sample size, inclusion of men and women, and data on several lifestyle factors and important confounders, including factors previously found to be related to mortality. The long and almost complete followup of the cohort resulted in a large number of outcome events.

Previous studies have identified lifestyle factors that promote health and increase longevity, including absence of current smoking, drinking a moderate amount of alcohol, participating in moderate exercise, and being of normal body mass index. However, few studies have investigated the combined effect of these lifestyle factors and even fewer have included the very old or, if they did, did not show agestratified results. In the HALE Project of European subjects aged 70 to 90 years, adherence to a Mediterranean diet (HR $=0.71)$ and healthful lifestyle (moderate alcohol use $(\mathrm{HR}=$ $0.74)$, physical activity $(\mathrm{HR}=0.65)$, and nonsmoking ( $\mathrm{HR}$ $=0.68)$ ) was associated with a lower rate of cardiovascular mortality [16]. In the Nurses' Health Study of middle-aged women, those who did not smoke cigarettes, were not overweight, maintained a healthful diet, exercised moderately or vigorously for half an hour a day, and consumed alcohol moderately had an incidence of coronary events that was more than 80 percent lower than that in the rest of the population [17]. In the SENECA (Survey in Europe on Nutrition and the Elderly: A Concerted Action) study of those aged 70-75 years, a high-quality diet, nonsmoking, and physical activity were positively related to 10 -year survival in both men and women [18]. For men, the mortality risk for a low-quality diet was 1.25 , for inactivity was 1.36 , and for smoking was 2.06. For women, the mortality risk for smoking was 1.76 and for inactivity 1.75 , much higher than the risk associated with a low-quality diet 1.26 . In the NHANES I Epidemiologic Followup Study, smoking predicted survival in middle-aged (45-54 years old) and older (65-74 years old) men and middle-aged women; nonrecreational physical activity predicted survival in older men and women; low body mass index was also associated with shorter survival in older men and middle-aged and older women; drinking was associated with shorter survival in older men [19].

Experimental, clinical, and epidemiological studies suggest mechanisms that provide a biological basis for causal relations between these behavioral risk factors and lower rates of CVD and death. Alcohol increases high-density lipoprotein cholesterol concentrations, decreases platelet aggregation, and affects tissue plasminogen activator and other components of clotting and fibrinolysis [3]. Likewise, physical activity reduces blood pressure, increases high-density lipoprotein cholesterol, decreases triglycerides, improves cardiorespiratory fitness, and produces beneficial changes in inflammatory/hemostatic factors [4].

The reason for increased mortality among the underweight elderly is not clear. Previous all-cause and CVD mortality studies in the elderly have found persons in the lowest weight category at increased risk of death [20,21]. Being lean, especially in the elderly, may represent a real risk because of nutrient deficiency and physical, functional, and psychological impairment. A balanced and healthful diet may be difficult for some elderly to maintain.

Evidence from epidemiologic studies indicates that the same factors that are associated with increased risk of CVD in middle-aged people are relevant in older adults. Although much effort has focused on the pharmacologic management of hypertension and blood lipid levels with proven success, lifestyle can also affect CVD mortality. Changing these risk factors in older adults can help reduce CVD risk as it does in middle-aged adults and without side effects, high cost, or medical intervention. Together avoidance of smoking, sensible drinking habits, regular physical activity, and maintenance of a healthy body weight may prevent much of the CVD in Western populations. With increasing age, the elderly, however, may become limited by comorbid conditions, decreased functional ability, impaired cognition, and emotional instability and therefore need special programs providing increased physical and social activities and balanced and healthful nutrition. Of course, the greatest benefit will be achieved by adopting these habits early in life and maintaining them throughout the life course.

\section{Conclusion}

Results in this large elderly cohort with long followup showing a decreased risk of cardiovascular mortality with 
several lifestyle practices suggest that maintenance of these is an important health promoter in aging populations.

\section{Acknowledgment}

This research was funded by Grants from the National Institutes of Health (R01CA32197 and R01AG21055), the Earl Carroll Trust Fund, and Wyeth-Ayerst Laboratories.

\section{References}

[1] D. Lloyd-Jones, R. J. Adams, T. M. Brown et al., "Executive summary: heart disease and stroke statistics-2010 update: a report from the American Heart Association," Circulation, vol. 121, no. 7, pp. e46-e215, 2010.

[2] A. H. Mokdad, J. S. Marks, D. F. Stroup, and J. L. Gerberding, "Actual causes of death in the United States, 2000," Journal of the American Medical Association, vol. 291, no. 10, pp. 12381245, 2004.

[3] R. A. Vogel, "Alcohol, heart disease, and mortality: a review," Reviews in Cardiovascular Medicine, vol. 3, no. 1, pp. 7-13, 2002.

[4] D. M. Buchner, "Physical activity and prevention of cardiovascular disease in older adults," Clinics in Geriatric Medicine, vol. 25, no. 4, pp. 661-675, 2009.

[5] A. Paganini-Hill, C. H. Kawas, and M. M. Corrada, "Type of alcohol consumed, changes in intake over time and mortality: the Leisure World Cohort Study," Age and Ageing, vol. 36, no. 2, pp. 203-209, 2007.

[6] A. Paganini-Hill, C. H. Kawas, and M. M. Corrada, "Nonalcoholic beverage and caffeine consumption and mortality: the Leisure World Cohort Study," Preventive Medicine, vol. 44, no. 4, pp. 305-310, 2007.

[7] National Heart, Lung, and Blood Institute, "Clinical guidelines on the identification, evaluation, and treatment of overweight and obesity in adults: the evidence report," Tech. Rep. 98-4083, National Heart, Lung, and Blood Institute, Bethesda, Md, USA, 1998, http://www.nhlbi.nih.gov/guidelines/obesity/ob_ gdlns.pdf.

[8] M. M. Corrada, C. H. Kawas, F. Mozaffar, and A. PaganiniHill, "Association of body mass index and weight change with all-cause mortality in the elderly," American Journal of Epidemiology, vol. 163, no. 10, pp. 938-949, 2006.

[9] D. R. Cox, "Regression models and life tables (with discussion)," Journal of the Royal Statistical Society B, vol. 34, pp. 187-220, 1972.

[10] A. Paganini-Hill and R. K. Ross, "Reliability of recall of drug usage and other health-related information," American Journal of Epidemiology, vol. 116, no. 1, pp. 114-122, 1982.

[11] A. Paganini-Hill, R. K. Ross, and B. E. Henderson, "Prevalence of chronic disease and health practices in a retirement community," Journal of Chronic Diseases, vol. 39, no. 9, pp. 699-707, 1986.

[12] A. Paganini-Hill, A. Chao, R. K. Ross, and B. E. Henderson, "Exercise and other factors in the prevention of hip fracture: the Leisure World Study," Epidemiology, vol. 2, no. 1, pp. 1625, 1991.

[13] A. Paganini-Hill and A. Chao, "Accuracy of recall of hip fracture, heart attack, and cancer: a comparison of postal survey data and medical records," American Journal of Epidemiology, vol. 138, no. 2, pp. 101-106, 1993.
[14] A. Paganini-Hill, "Risk factors for Parkinson's disease: the Leisure World Cohort Study," Neuroepidemiology, vol. 20, no. 2, pp. 118-124, 2001.

[15] A. Paganini-Hill and G. Hsu, "Smoking and mortality among residents of a California retirement community," American Journal of Public Health, vol. 84, no. 6, pp. 992-995, 1994.

[16] K. T. B. Knoops, L. C. P. G. M. de Groot, D. Kromhout et al., "Mediterranean diet, lifestyle factors, and 10-year mortality in elderly European men and women: The HALE project," Journal of the American Medical Association, vol. 292, no. 12, pp. 1433-1439, 2004.

[17] M. J. Stampfer, F. B. Hu, J. E. Manson, E. B. Rimm, and W. C. Willett, "Primary prevention of coronary heart disease in women through diet and lifestyle," The New England Journal of Medicine, vol. 343, no. 1, pp. 16-22, 2000.

[18] A. Haveman-Nies, L. P. G. M. de Groot, J. Burema, J. A. Amorim Cruz, M. Osler, and W. A. Van Staveren, "Dietary quality and lifestyle factors in relation to 10-year mortality in older Europeans: the SENECA Study," American Journal of Epidemiology, vol. 156, no. 10, pp. 962-968, 2002.

[19] M. A. Davis, J. M. Neuhaus, D. J. Moritz, D. Lein, J. D. Barclay, and S. P. Murphy, "Health behaviors and survival among middle-aged and older men and women in the NHANES I Epidemiologic Follow-up Study," Preventive Medicine, vol. 23, no. 3, pp. 369-376, 1994.

[20] K. M. Flegal, B. I. Graubard, D. F. Williamson, and M. H. Gail, "Excess deaths associated with underweight, overweight, and obesity," Journal of the American Medical Association, vol. 293, no. 15, pp. 1861-1867, 2005.

[21] J. Stevens, J. Cai, E. R. Pamuk, D. F. Williamson, M. J. Thun, and J. L. Wood, "The effect of age on the association between body-mass index and mortality," The New England Journal of Medicine, vol. 338, no. 1, pp. 1-7, 1998. 


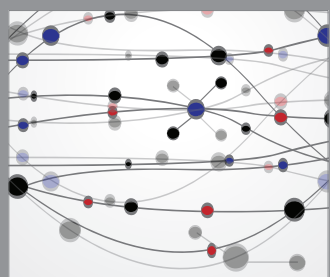

The Scientific World Journal
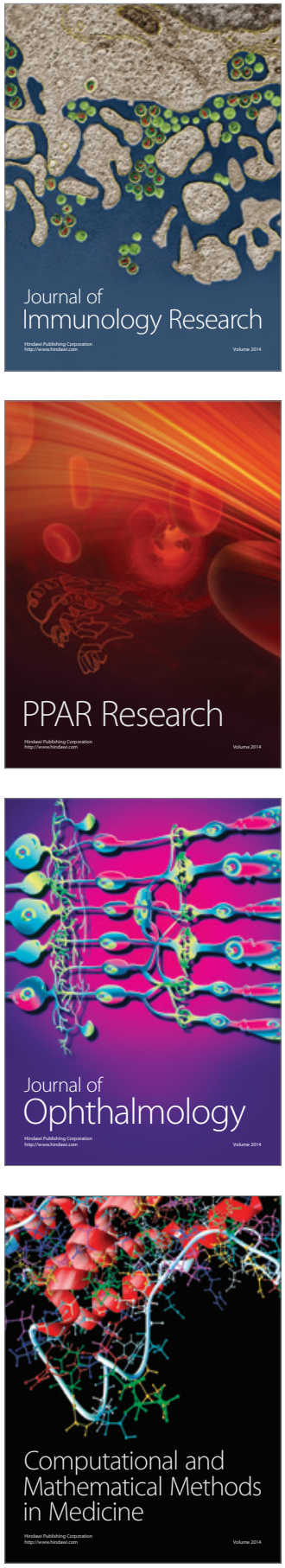

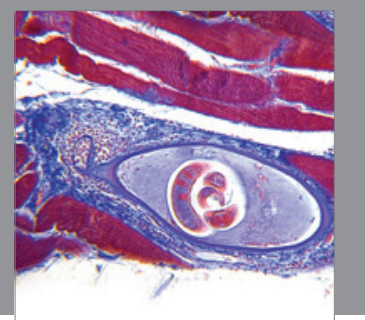

Gastroenterology

Research and Practice
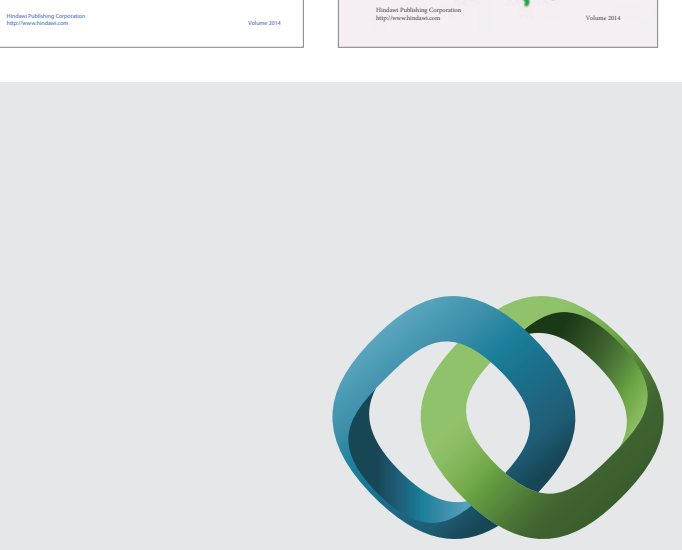

\section{Hindawi}

Submit your manuscripts at

http://www.hindawi.com
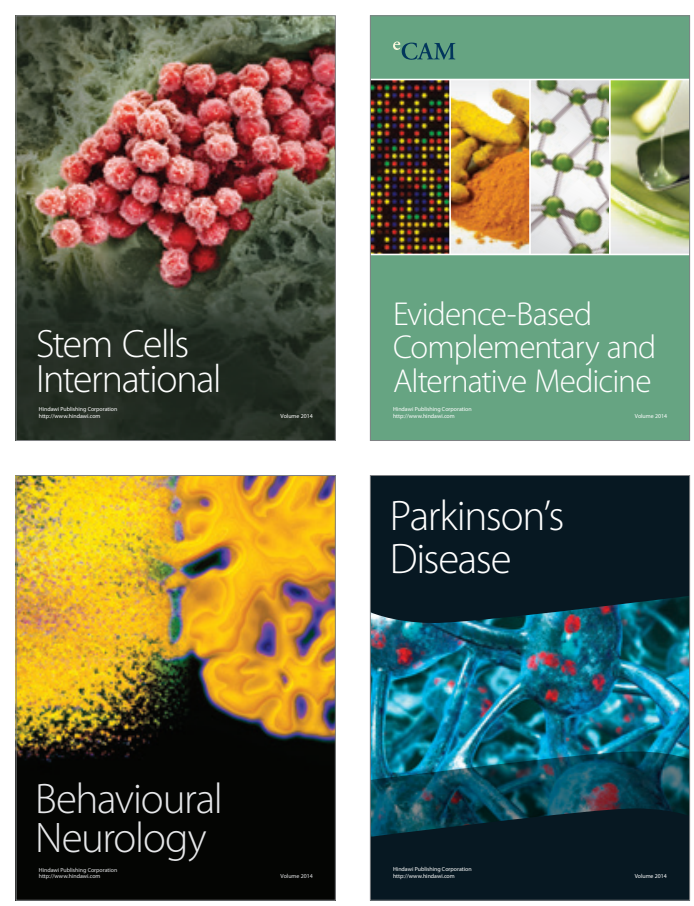

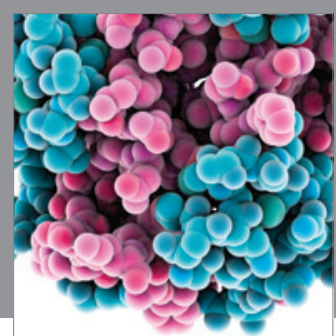

Journal of
Diabetes Research

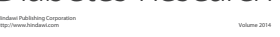

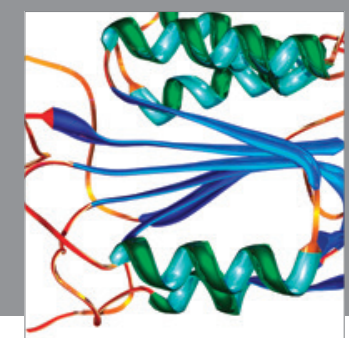

Disease Markers
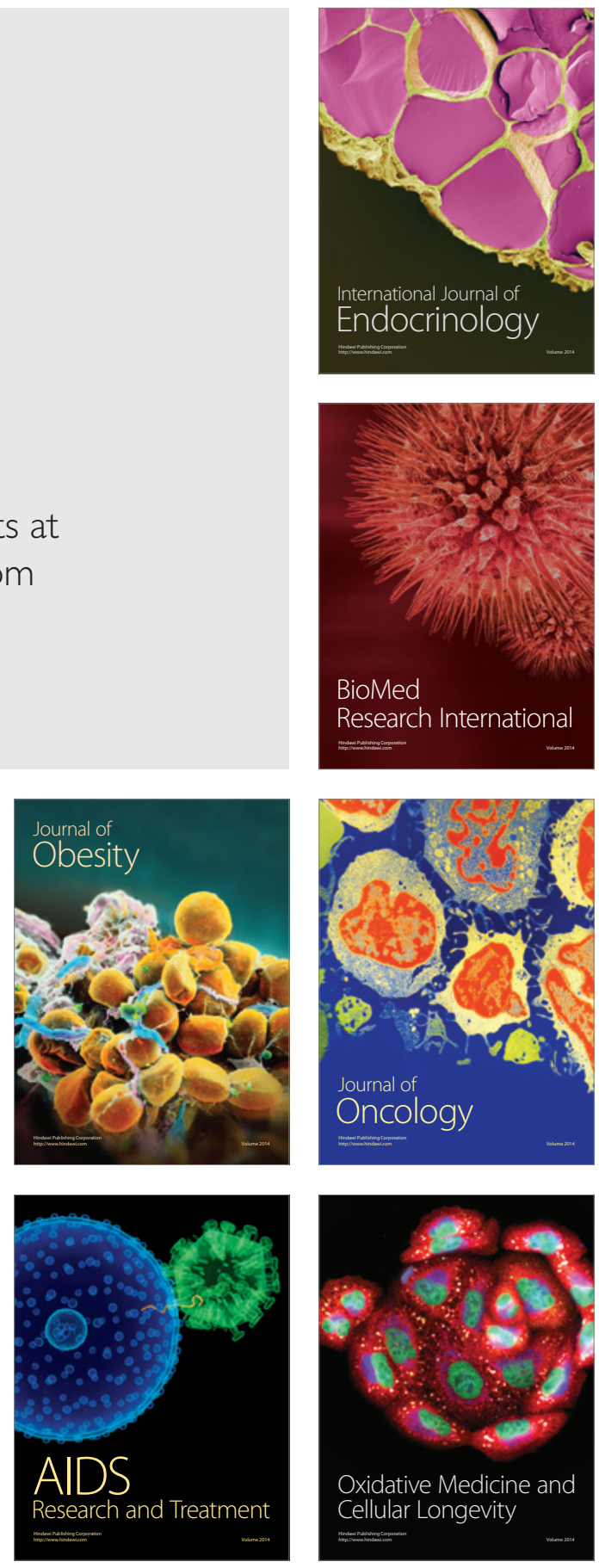